\title{
Liquid crystal tunable photonic crystal dye laser
}

\author{
Buss, Thomas; Christiansen, Mads Brøkner; Smith, Cameron; Kristensen, Anders
}

Published in:

2010 Conference on Lasers and Electro-Optics (CLEO) and Quantum Electronics and Laser Science Conference (QELS)

Publication date:

2010

Document Version

Publisher's PDF, also known as Version of record

Link back to DTU Orbit

Citation (APA):

Buss, T., Christiansen, M. B., Smith, C., \& Kristensen, A. (2010). Liquid crystal tunable photonic crystal dye laser. In 2010 Conference on Lasers and Electro-Optics (CLEO) and Quantum Electronics and Laser Science Conference (QELS) (pp. 1-2). IEEE.

\section{General rights}

Copyright and moral rights for the publications made accessible in the public portal are retained by the authors and/or other copyright owners and it is a condition of accessing publications that users recognise and abide by the legal requirements associated with these rights.

- Users may download and print one copy of any publication from the public portal for the purpose of private study or research.

- You may not further distribute the material or use it for any profit-making activity or commercial gain

- You may freely distribute the URL identifying the publication in the public portal

If you believe that this document breaches copyright please contact us providing details, and we will remove access to the work immediately and investigate your claim. 


\title{
Liquid crystal tunable photonic crystal dye laser
}

\author{
Thomas Buß, Mads B. Christiansen, Cameron L.C. Smith, Anders Kristensen \\ Department of Micro- and Nanotechnology, DTU Nanotech, Technical University of Denmark, DK-2800 Kongens Lyngby, Denmark \\ Corresponding author: anders.kristensen@nanotech.dtu.dk; Phone: +45-4525 6331,Fax: +45-4588 7762
}

\begin{abstract}
We present a dye-doped liquid crystal laser using a photonic crystal cavity. An applied electric field to the liquid crystal provides wavelength tunability. The photonic crystal enhances resonant interaction with the gain medium.

(C) 2010 Optical Society of America

OCIS codes: (230.5298) Photonic crystals; (230.3720) Liquid-crystal devices
\end{abstract}

\section{Introduction}

Optofluidic lasers $[1,2]$ are promising devices for affordable integration of light sources onto lab-on-a-chip systems [3]. The tunability of optofluidic lasers is particularly desirable for optical sensing applications, where there have been several demonstrations tuning microfluidic dye lasers by means of changing the dye concentration [4] or mixing different dyes [5]. However, these microfluidic systems suffer from complex fluid supply networks and slow time responses. Liquid crystals (LCs) offer a suitable approach to circumvent these issues by providing electrical tunability via their large electro-optic interaction and dyes may be dissolved in the LC to allow optical gain. One such LC device has been presented using holographic excitation to form a DFB cavity [6], yet in this case the Bragg grating offers dispersion control in only 1D. Photonic crystals (PhCs) offer much greater control of light by engineering their dispersion in 2D or 3D [7]. Accordingly, a 2D PhC with polymer-dispersed LC has been recently demonstrated by using holography [8] but without exploiting the LC tunability. Holography is an elegant method to fabricate sub-micrometer structures, although the design freedom is limited. E-beam and Nano-imprint lithography allow the fabrication of arbitrary structures; a tunable LC laser fabricated by imprinting a Bragg grating into a polymer has recently been shown [9]. In the current work we extend this type of laser, demonstrating electrically induced wavelength tunability together with a multifunctional $\mathrm{PhC}$ resonator. The $\mathrm{PhC}$ efficiently enhances the gain interaction $50 \mathrm{~nm}$ away from the dye fluorescence maximum.

\section{Tunable photonic crystal laser concept}

Device schematics are shown in Fig. 1 (a) and (b), illustrating the top and side views. The SU-8 spacers (320 nm thickness) define the height between the PhC and upper indium tin oxide (ITO) coated glass cover to freely enable electrically induced orientation of the LC molecules.

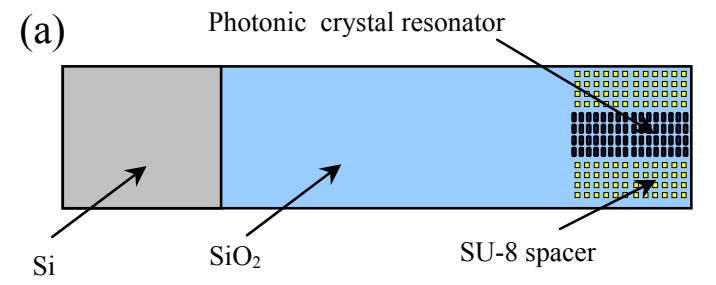

(b)

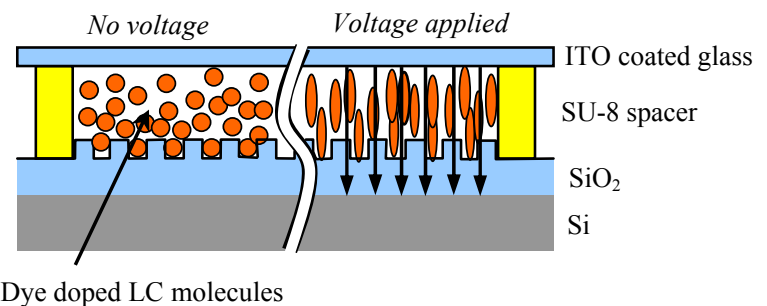

(d)

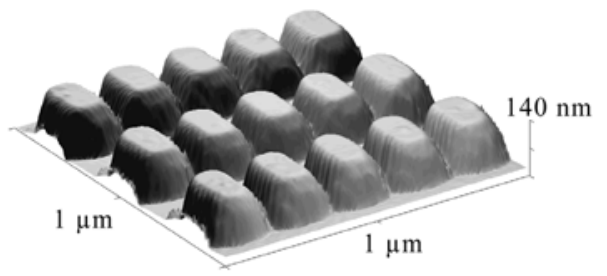

Figure 1. (a) Top view and (b) side view of a schematic drawing of the PhC LC laser chip. LC molecule alignment is illustrated without / with applied voltage. (c) SEM image of the PhC resonator with 361 x $200 \mathrm{~nm}$ unit cell. (d) AFM scan of the PhC. The height of the PhC is $140 \mathrm{~nm}$. 


\section{CWM6.pdf}

Initially the nematic phase LC molecules align along the grooves of the $\mathrm{PhC}$ structure and tilt towards vertical alignment under an applied E-field. This causes a refractive index change of the cavity modes in the $\mathrm{PhC}$ and results in a wavelength shift of the laser emission.

The PhC lasers can be tailored to both define their lasing emission wavelengths as well as increase the coupling of pump light to the laser cavities, significantly lowering lasing thresholds [10]. The devices in this work use rectangular lattice $\mathrm{PhC}$ resonators with a dye doped liquid crystal gain medium infiltrating the $\mathrm{PhC}$. The $\mathrm{LC}$ is $5 \mathrm{CB}$, which is doped with the laser dye Pyrromethene 597 (1 wt \%).

Figure 1 (c) and (d) displays SEM and AFM images of the PhC sections. A unit cell of the $\mathrm{PhC}$ has dimensions $361 \times 200 \mathrm{~nm}$, where the shorter grating forms the laser cavity and the larger couples the pump light to a cavity mode. The depth of the $\mathrm{PhC}$ pattern is $140 \mathrm{~nm}$. The lasers are fabricated on a silicon wafer with a $4.5 \mu \mathrm{m}$ oxide layer, using E-beam lithography to define the PhC geometry. In a subsequent lithography step the SU-8 spacers are exposed. Some $\mathrm{SiO}_{2}$ is removed at the end of the device to allow electrical contact to the silicon (bottom electrode). A drop of laser dye is placed on the $\mathrm{PhC}$ and then covered by the ITO coated glass plate (top electrode).

\section{Optical Characterization}

Pumping of the lasers is done through the transparent top electrode using a frequency doubled Nd:YAG laser, emitting $10 \mathrm{~ns}$ pulses with an energy of $200 \mu \mathrm{J}$. The dye laser emission is observed in figure 2. Panel (a) shows the emission when the pump laser angle is not resonant with the $\mathrm{PhC}$ long lattice direction. Accordingly, laser emission occurs at the fluorescence maximum. Panel (b) and (c) show laser emission with the resonant pump angle, forcing the laser emission to the wavelength defined by the PhC. Furthermore a clear electrically induced wavelength shift is observed when voltage is applied. The lasing peaks occur $50 \mathrm{~nm}$ away from the dye fluorescence maximum, suggesting high lasing efficiencies and broad device flexibility. The ratio of the laser peaks to the fluorescence maximum is over $10 \mathrm{~dB}$, comparing favorably with other similar 1D grating devices [9] and showing that the 2D $\mathrm{PhC}$ enhances the resonant pump interaction with the dye gain medium.
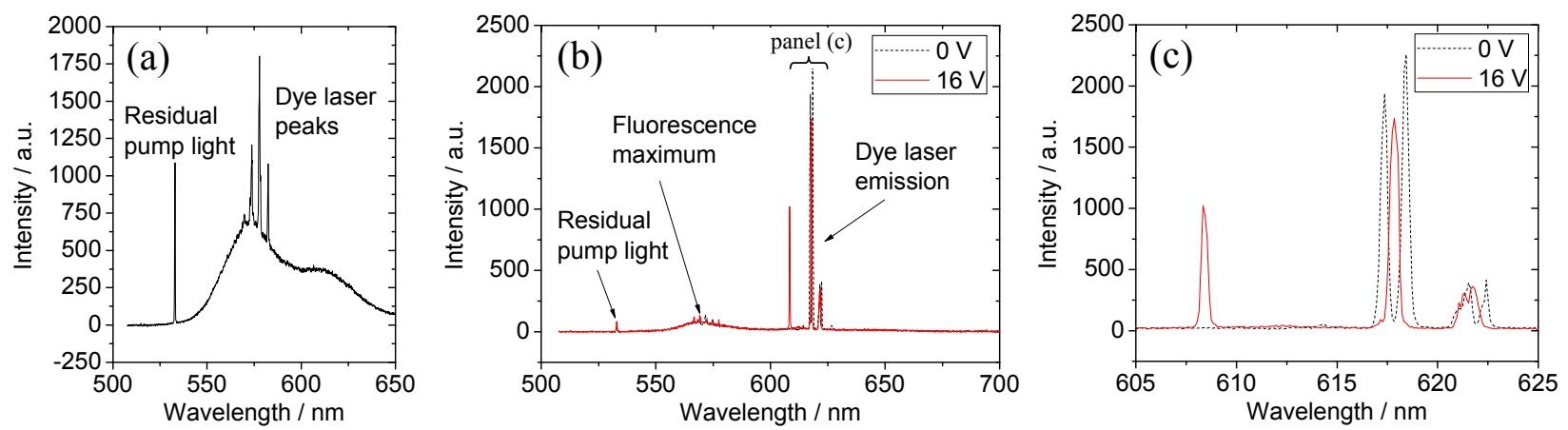

Figure 2. Device emission spectra. (a) Emission with de-tuned pump angle (b) Spectrum with matched pump angle (c) Close-up of the lasing peaks. The black (dotted) trace shows the spectrum without applied voltage, the red (solid) trace the shifted spectrum while voltage is applied.

\section{Conclusion}

In conclusion, we present a tunable laser with a multifunctional $\mathrm{PhC}$ resonator. The tunability is achieved by incorporating $\mathrm{LC}$ to a $\mathrm{PhC}$ device and applying an E-field. The PhC strongly enhances pump interaction with the gain medium. This work is highly relevant for the advancement of optical analysis in lab-on-a-chip systems.

1. B. Helbo, A. Kristensen, and A. Menon, "A micro-cavity fluidic dye laser," J. Micromech. Microeng. 13, 307-311 (2003).

2. C. Monat, P. Domachuk, and B. J. Eggleton, "Integrated optofluidics: A new river of light," Nat. Photonics 1, 106-114 (2007).

3. D. Janasek, J. Franzke, and A. Manz, "Scaling and the design of miniaturized chemical-analysis systems," Nature 442, 374-380 (2006).

4. B. Bilenberg, T. Rasmussen, S. Balslev, and A. Kristensen, "Real-time tunability of chip-based light source enabled by microfluidic mixing," J. Appl. Phys. 99, 5 (2006).

5. Z. Li and D. Psaltis, "Optofluidic Distributed Feedback Dye Lasers," IEEE Journal of Selected Topics in Quantum Electronics 13, 185-193 (2007).

6. T. Matsui, M. Ozaki, and K. Yoshino, "Tunable laser action in a dye-doped nematic liquid-crystal waveguide under holographic excitation based on electric-field-induced TM guided-mode modulation", J. Opt. Soc. Am. B-Opt. Phys. 21, 1651-1658 (2004).

7. J. D. Joannopoulos, R. D. Meade and J. N. Winn, "PhotonicCrystals: Molding the Flow of Light," Princeton University Press, 1995.

8. D. Lou, X. W. Sun, H. T. Dai, Y. J. Liu, H. Z. Yang, and W. Ji, "Two-directional lasing from a dye-doped two-dimensional hexagonal photonic crystal made of holographic polymer-dispersed liquid crystals," Appl. Phys. Lett. 95, 151115 (2009).

9. R. Ozaki, T. Shinpo, K. Yoshino, M. Ozaki and H. Moritake, "Tunable liquid crystal laser using distributed feedback cavity fabricated by nanoimprint lithography," Applied Physics Express 1, 012003 (2008).

10. M. B. Christiansen, A. Kristensen, S. S. Xiao, and N. A. Mortensen, "Photonic integration in k-space: Enhancing the performance of photonic crystal dye lasers," Appl. Phys. Lett. 93, 3 (2008). 